\title{
REPENSAR LOS CONFLICTOS INTERCULTURALES Y SU TRANSFORMACIÓN PACÍFICA DESDE EL PARADIGMA DE LA COMPLEJIDAD DE HERÁCLITO DE ÉFESO
}

\author{
RETHINKING THE INTERCULTURAL CONFLICTS AND THEIR \\ PEACEFUL TRANSFORMATION FROM THE PARADIGM OF \\ COMPLEXITY BY HERACLITUS OF EPHESUS
}

\author{
Sonia París Albert ${ }^{1}$ \\ Universitat Jaume I (España)
}

Recibido: 30-11-2014

Aceptado: 02-02-2015

Resumen: El pensamiento del filósofo presocrático Heráclito de Éfeso es una herramienta muy útil para el estudio de los conflictos interculturales y su posible regulación por medios pacíficos. Este es el motivo por el que en estas páginas se analizarán algunas de las características de su filosofía, especialmente aquellas que están en relación con lo que aquí hemos venido a denominar su paradigma de la complejidad. Este análisis se realizará, principalmente, con el propósito de seguir profundizando en la investigación sobre los conflictos interculturales, así como sobre las posibles alternativas para su transformación pacífica.

Palabras-clave: Heráclito de Éfeso, paradigma de la complejidad, conflictos interculturales y transformación pacífica de conflictos.

\begin{abstract}
The thought by the Pre-Socratic Philosopher Heraclitus of Ephesus is a very useful tool to study the intercultural conflicts and their possible regulation by peaceful means. This is the reason why, in these pages, some of the characteristics of his Philosophy, especially those, which are related to what we have come to call the paradigm of complexity, will be analyzed. This analysis will be done, mainly, in order to further deepen in the research on intercultural conflicts as well as in the possible alternatives for their peaceful transformation.
\end{abstract}

Key-words: Heraclitus of Ephesus, paradigm of complexity, intercultural conflicts and peaceful conflict transformation.

[1] (sparis@uji.es) Doctora por la Universitat Jaume I (Castellón, España) y profesora de Filosofía en el Departamento de Filosofía y Sociología de la misma universidad. Actualmente, es Vice-Directora de la Cátedra UNESCO de Filosofía para la Paz y Coordinadora del Máster Universitario en Estudios Internacionales de Paz, Conflictos y Desarrollo de la Universitat Jaume I. 


\section{Introducción ${ }^{2}$}

Realizar un estudio de los conflictos y su posible transformación pacífica a partir de las filosofías de pensadores clásicos y modernos es una tarea que tenía pendiente desde hace algún tiempo y parece que, por fin, he podido empezar a abordarla en este trabajo. En este sentido, las siguientes páginas se presentan con el propósito de analizar algunas de las más destacables características de la cosmovisión del mundo de Heráclito de Efeso, que son, al mismo tiempo, las que pueden ayudarnos a interpretar las situaciones conflictivas. Por esta razón, los primeros apartados de este texto nos aproximarán al pensamiento del filósofo presocrático de Efeso para, a continuación, resumir algunos rasgos de los conflictos y su transformación pacífica en relación con esta filosofía. El objetivo, entonces, será que este pensador clásico nos ayude a seguir profundizando, desde lo que vendremos a calificar como su paradigma de la complejidad, tanto en la concepción de los conflictos cómo en la metodología para su transformación pacífica que venimos trabajando en la filosofía para la paz de la Cátedra UNESCO de Filosofía para la Paz de la Universitat Jaume I de Castellón, aunque sin la pretensión de dar una descripción completa de sus rasgos, sino sólo destacando aquellos que se pueden poner en conexión con la filosofía compleja de Heráclito.

Observamos, por consiguiente, que no se trata de establecer relaciones entre diferentes pensadores clásicos y modernos, sino que concentraremos nuestra atención en la filosofía de Heráclito. Además, también es importante mencionar que este estudio se referirá, de forma concreta, a los conflictos interculturales, a pesar de que los rasgos señalados puedan ser aplicables a cualquier otra clase de conflictos.

Finalmente, cabe indicar, como ya se ha dejado entrever previamente, que este trabajo se enmarca en el conjunto de las investigaciones sobre conflictos interpersonales que he venido realizando, durante los últimos años, dentro del grupo de investigación de filosofía para la paz de la Universitat Jaume I de Castellón. Grupo que viene siendo ampliamente reconocido en los estudios para la paz tanto a nivel nacional como internacional.

[2] Este trabajo se inserta en el proyecto de investigación "De víctimas a indignados: visibilidad mediática, migración de imágenes, espectacularización de los conflictos y procesos de transformación social hacia una cultura de paz" (código P1·1A2012-05), dirigido por el Dr. Vicente José Benet Ferrando del Departamento de Ciencias de la Comunicación de la Universitat Jaume I, y financiado por el Plan de Promoción de la Investigación de la misma universidad. 


\section{Heráclito de Efeso: un filósofo complejo, un pensador apodado El Oscuro}

Nacido en Efeso, Heráclito ha pasado a ser uno de los pensadores presocráticos más destacables de la Antigüedad, no sólo por los datos que tenemos de su filosofía, sino, también y muy especialmente, por su carácter complejo. Así y de acuerdo con el sentido general de la filosofía presocrática, que era el de indagar el principio último (arjé) a partir del cual se originaban todos los restantes elementos de la realidad, Heráclito se propuso dibujar una imagen del mundo, aunque, en esta ocasión y según Spengler ${ }^{3}$, sería ésta una nueva y diferente cosmovisión del mundo ${ }^{4}$. Hasta tal punto es así que Spengler señala que, en lo que a su pensamiento se refiere, Heráclito puede ser conocido como un psicólogo, pues lo que en él se encuentra es, ciertamente, una psicología de los acontecimientos del mundo. De todas maneras, el estudio de su obra no es una tarea sencilla, ya que a la complejidad de su pensamiento, se añade el hecho de haberse perdido gran parte de sus textos, hecho que hace todavía más difícil disponer de una visión completa y única de este pensador, pues en la medida que se van leyendo las investigaciones acerca de él publicadas, se va haciendo evidente la gran variedad de interpretaciones que sobre su filosofía existen ${ }^{5}$.

$\mathrm{Si}$ en lo que a su pensamiento se refiere puede ser conocido como un psicólogo, en su vida cotidiana se identificaba plenamente con la aristocracia, de manera que "[...] quería la diferencia entre mandador y mandado, honraba las costumbres transmitidas por la antigüedad y sus instituciones [...]» $»^{6}$. Es decir, Heráclito era ampliamente partidario de mantener el ideal de vida en el que él mismo había sido educado, pero ya en aquellos tiempos, cuando él había alcanzado la madurez en las LXIX Olimpiadas (504-501) ${ }^{7}$, estaba teniendo lugar el nacimiento de la democracia y, por consiguiente, una nueva forma de organización social y política se estaba instaurando, la cual, por encima de todo, impedía el mantenimiento de las estructuras clásicas. Estos cambios, junto con su personalidad tosca e irritante, hicieron que Heráclito se refugiara en los

[3] Spengler, O.: Heráclito. Sevilla: Ediciones Espuela de Plata, 2013, p. 116.

[4] En mi opinión, sería ésta una nueva y diferente cosmovisión del mundo porque, entre otras cosas, no podemos decir que Heráclito estableciese un primer principio (arjé) en su interpretación de la realidad, ya que el elemento fuego adopta, más bien, en su filosofía el sentido de metáfora del mundo, tal y como algunas investigaciones señalan (Cf. Ibídem). Sin embargo, también es importante tener en cuenta que otros estudios, con líneas de pensamiento diferentes, sí consideran el elemento fuego como primer principio en Heráclito.

[5] Ibídem.

[6] Ibídem, p. 112.

[7] Gallero, J. L. y López, C. E.: Heráclito: fragmentos e interpretaciones. Madrid: Ardora Ediciones, 2009.

THÉMATA. Revista de Filosofía, №52 julio-diciembre (2015) pp.: 179-197

doi: 10.12795/themata.2015.i52.10 
montes y que llevara a cabo un estilo de vida que lo hizo caer, prácticamente, en la locura ${ }^{8}$.

Esta forma de vida, junto con su carácter y la complejidad de su pensamiento provocaron que Cicerón lo apodara como El Oscuro, un apodo al que, también, contribuyó su particular manera de escribir, muy similar a la de los poetas, aunque hay que decir que nunca llegó a redactar en verso ${ }^{9}$.

Heráclito de Efeso fue, entonces, un pensador presocrático, tremendamente avanzado en sus ideas para los tiempos en los que vivió y, muy pronto, entiendo que identificado como distante a la tradición occidental, una tradición, desde siempre, encantada con la búsqueda de principios universales para dar explicación a las cosas, a los hechos, al mundo... Ha influenciado en filósofos diversos como el mismo Nietzsche ${ }^{10}$, quien, también frente a la tradición occidental, defendió el mundo de la vida con su propuesta del superhombre y una filosofía basada en la corriente del vitalismo. En su caso, Heráclito, tal y como se observará en los siguientes apartados, propondrá, desde lo que en estas páginas vendremos a llamar un paradigma de la complejidad, la importancia del devenir, el cambio y el movimiento, a modo de reconocimiento del mundo dinámico y del flujo perpetuo frente, entiendo, al estatismo propuesto con principios universales inamovibles por la tradición occidental, aunque sin que por ello creo que debamos pensar que ejerce una defensa enraizada de los sentidos, pues no podemos olvidar que para este autor, y como él mismo afirma, «la armonía invisible es superior a la visible» ${ }^{11}$. En las siguientes páginas de este texto trataremos de adentrarnos en gran parte de estos conceptos e interpretaciones.

\section{Oposición y devenir: rasgos distintivos del paradigma de la complejidad en la filosofía de Heráclito}

Cuando Heráclito presenta su cosmovisión del mundo parte del ras-

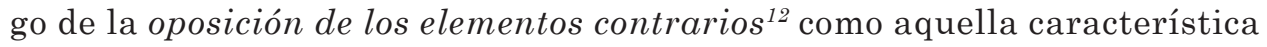

[8] Spengler, O.: op cit.

[9] Gallero, J. L. y López, C. E.: op. cit.

[10] Colomina Alminyana, J. J.: «Nietzsche y Heráclito» en ÉNDOXA: Series Filosóficas 23, 2009, pp. 177-190.

[11] Montes, Á. C.: Repensar a Heráclito. Madrid: Ed. Trotta, 2011, p. 217.

[12] Los siguientes pueden considerarse tipos de oposiciones: «1) Cosas que producen efectos opuestos sobre clases distintas de seres animados, como el agua del mar, saludable para los peces, pero no para los seres humanos. 2) Aspectos diferentes de la misma cosa pueden justificar descripciones opuestas, como un camino, que aunque se recorra hacia arriba o hacia abajo no deja de ser el mismo, sin ser lo mismo hacerlo en un sentido u en otro. 3) Algunas realidades solo son concebibles por medio de sus opuestos, como la salud que no sería agradable si no existiera la enfermedad. 4) Algunos opuestos están enlazados de un modo esencial, siendo dos aspectos distintos y sucesivos de una misma realidad: lo caliente y lo frío» (Cf. Fernández Pérez, G.: Heráclito.

THÉMATA. Revista de Filosofía, N52 julio-diciembre (2015) pp.: 179-197 doi: 10.12795/themata.2015.i52.10 
que se convierte en el eje central de toda su filosofía ${ }^{13}$. Tanto es así que para este filósofo presocrático todo en la naturaleza ${ }^{14}$ se encuentra en una continua tensión, siendo posible conocer las cosas que forman el mundo al tener todas ellas un opuesto a partir del cual se configuran y se constituyen. Opuestos que se encuentran en conexión si tomamos en consideración una de sus sentencias, según la cual "es sabio convenir que todas las cosas son una» ${ }^{1516}$. La trascendencia que el rasgo de la oposición adquiere en la filosofía de este pensador se puede observar en las siguientes citas textuales:

«(22 B 80) oRíg., C. Celso VI 42: Al decir Heráclito « es necesario saber que la guerra es común y la justicia discordia, y que todo sucede según discordia y necesidad» ${ }^{17}$.

«Es necesario saber que la contienda es universal y la discordia justicia, y todo se genera por discordia y necesidad ${ }^{18}$.

«La lucha de los opuestos es lo único real en el incesante devenir que es como las aguas de un río que siempre se renuevan» ${ }^{19}$.

«[...] todo pivota entorno al conflicto permanente» ${ }^{20}$.

El valor de la oposición no sólo se vincula con el mundo material en la filosofía de Heráclito, sino también con la naturaleza humana. Esta es la razón por la que el autor considera que cuando las personas se contemplan

Naturaleza y complejidad. Sevilla: Ed. Thémata, 2010, pp. 125-126). Además, podemos añadir según la interpretación de este texto: 5) Perspectivas, puntos de vista y/o posiciones contrapuestas. 6) Pensamientos y acciones diferentes, etc.

[13] García Junceda, J.A.: «Uno y múltiple: La dialéctica de los contrarios en Heráclito» en Anales del Seminario de Historia de la Filosofía 4, 1984, pp. 29-44.

[14] El concepto de naturaleza tiene sentidos diversos en la filosofía presocrática (Cf. Fernández Pérez, G.: op. cit., p. 74). Por ejemplo, (1) puede aludir a todo aquello que hay, (2) al conjunto de cualidades que hacen que una cosa sea lo que es y/o (3) al primer principio (arjé) del que nacen todas las cosas y al que ya nos hemos referido en el segundo apartado de este texto al mencionar el sentido general de la filosofía presocrática. De todas maneras, en Heráclito cabe tener en cuenta que se hace referencia al concepto de naturaleza en sentido estricto (Cf. Ibídem, p. 88), aunando en su filosofía el estudio de la naturaleza material con el de la naturaleza humana (Cf. Ibídem, p. 87), tal y como se expondrá en este apartado.

[15] Montes, Á. C.: op. cit., p. 46.

[16] Pensadores como Spengler (Cf. Spengler, O.: op. cit., p. 45) niegan, en cambio, que Heráclito se refiera aquí a una identidad real de los opuestos, "atribuyéndole únicamente la aseveración de una identidad de forma entre ellos, considerados como antinomias, es decir en cuanto que ninguno de los dos puede existir sin su contrario, viviendo cada uno la muerte del otro».

[17] Eggers Lan, C. y Juliá, V. E.: Los filósofos presocráticos I. Madrid: Ed. Gredos, 1978, p. 347.

[18] Gallero, J. L. y López, C. E.: op. cit., p. 124.

[19] Spengler, O.: op. cit., p. 10.

[20] Montes, Á. C.: op. cit., p. 53.

THÉMATA. Revista de Filosofía, Nº52 julio-diciembre (2015) pp.: 179-197

doi: 10.12795/themata.2015.i52.10 
a sí mismas, observan, sin ninguna duda, que su interior, mayoritariamente, está cargado de posicionamientos encontrados y de indecisiones ${ }^{21}$, tal y como se observa en los siguientes extractos:

«La vida es, precisamente, esa misma pugna, porque todo lo que está vivo pelea [...]»²2.

«La vida humana es también el producto de una tensión interna entre elementos contrarios (opiniones, deseos, intereses, afectos y pasiones, etc.), que son particulares y deben ser armonizados en los común, por medio de la misma razón que impera y opera en la naturaleza» ${ }^{23}$.

«La naturaleza en su conjunto, en suma, incluyendo la vida humana, es a un tiempo lucha manifiesta y unidad latente, de modo que es la tensión la que ajusta el cosmos, cuya faz visible es una lucha perpetua, que a la postre se desvela, para la mente despierta, como un aspecto parcial de un entramado global e invisible que todo lo liga, en la que los opuestos coinciden» ${ }^{24}$.

Por consiguiente, las anteriores son un conjunto de citas que nos permiten aludir a la continua oposición que para Heráclito existe tanto en el mundo material como en el mental del ser humano ${ }^{25}$; oposición causante de incesantes conflictos que hacen pensar en la eterna tensión como, además, la única vía para alcanzar una posible reconciliación en el mundo en caso que la hubiera ${ }^{26}$, pues:

Si todos los hombres tuviéramos las mismas ideas, si éstas fueran, precisamente aquellas que imparte el poder arbitrario y si quedasen proscritos la discusión y el debate, el declinar intelectual del hombre estaría asegurado, su condición servil resultaría inevitable y su mejor atributo, la inteligencia libre, degeneraría inevitablemente $[\ldots]^{27}$.

Estas últimas palabras que se incluyen de Montes hacen pensar en la necesidad ineludible de esa continua tensión que hemos venido señalando en las líneas anteriores; de esos constantes conflictos que tienen lugar en el mundo tanto material como humano y que hacen evidente otro rasgo fundamental de la cosmovisión del mundo en Heráclito: la complejidad de la naturaleza ${ }^{28}$. La naturaleza es compleja porque en ella no todo sucede fácilmente, sino que los acontecimientos van pasando a medida que van encontrando obstáculos, que van entrando en contradicción los unos con los otros y que van viviendo en

[21] Ibidem, p. 49.

[22] Ibidem, p. 53.

[23] Fernández Pérez, G.: op. cit., p. 137.

[24] Ibidem, p. 127.

[25] Montes, Á. C.: op. cit., p. 47.

[26] Fernández Pérez, G.: op. cit., p. 157.

[27] Montes, Á. C.: op. cit., p. 51.

[28] Fernández Pérez, G.: op. cit., p. 22.

THÉMATA. Revista de Filosofía, N52 julio-diciembre (2015) pp.: 179-197 doi: 10.12795/themata.2015.i52.10 
una continua tensión con sus opuestos. Esa misma complejidad se encuentra en la naturaleza humana, la cual cargada de indecisiones y de pensamientos opuestos tiene que ir adoptando decisiones y afrontando las situaciones que le va presentado la vida. Sin embargo, la complejidad de la naturaleza material y humana no sólo viene dada por el rasgo de la oposición, sino también por otra de las características principales que Heráclito utiliza para diseñar su cosmovisión del mundo: el continuo devenir. Ni que decir tiene que con la introducción de este rasgo, Heráclito se convierte en uno de los primeros pensadores en hablar del movimiento y del cambio con la finalidad de ejemplificar ese constante fluir y dinamismo de la realidad material y humana. Con este sentido se redactan los siguientes párrafos:

«578 (22 A 6) PLATón, Crát. 402: En algún lugar dice Heráclito que todo se mueve y nada permanece, y, comparando las cosas con la corriente de un río, dice que en el mismo río no nos bañamos dos veces» ${ }^{29}$.

«A quienes entran en los mismos ríos bañan aguas siempre nuevas» ${ }^{30}$.

«Vemos el transcurrir del mundo como si estuviéramos sobre la ribera de un río; incesantemente fluye delante de nosotros, siempre igual, sin principio ni fin, sin causa o meta: Podemos comprender lo acontecido en el cosmos sólo según su carácter; no podemos abrazarlo con la vista en su totalidad, como acontecimiento» ${ }^{31}$.

"[...] la naturaleza es un proceso ígneo incesante que da lugar a todas las cosas sin perder por ello su mismidad, en un ciclo perpetuo de relaciones y retro-acciones donde cada movimiento es inédito incluso en su repetición, es decir, donde acontece el retorno temporal de lo mismo como otro» ${ }^{32}$.

Aún más. No sólo es uno de los filósofos pioneros de la investigación sobre el movimiento, sino, también, creo que uno de los primeros pensadores en apartarse de la corriente que, a partir de estos momentos y hasta la actualidad, caracterizará, en mayor medida, a la filosofía occidental. Con la inclusión del cambio y del movimiento, considero que Heráclito supera, al menos en cuanto al arjé se refiere, la incesante necesidad de Occidente de encontrar unos principios universales inamovibles, capaces de dar sentido y explicación a las cosas, estemos en los tiempos que estemos y en cualquier lugar del mundo. Desde Grecia, la filosofía occidental se ha esforzado por justificar los hechos, las cosas y el pensamiento con la existencia de unos universales que otorgasen un sentido estático al mundo y que permitiesen explicar conceptos, procedimientos y

[29] Eggers Lan, C. y Juliá, V. E.: op. cit., p. 326

[30] Gallero, J. L. y López, C. E.: op. cit., p. 44.

[31] Spengler, O.: op. cit., p. 139.

[32] Fernández Pérez, G.: op. cit., p. 88.

THÉMATA. Revista de Filosofía, Nº52 julio-diciembre (2015) pp.: 179-197 doi: 10.12795/themata.2015.i52.10 
conductas. Heráclito, en cambio, creo que evita al máximo hablar de la existencia de estas esencias, universales y/o principios últimos, al menos en cuanto a la búsqueda de un arjé estático, pues en su cosmovisión del mundo natural y humano todo es flujo perpetuo, siendo así imposible encontrar principios estables aplicables a todo y a todos los sujetos ${ }^{33}$. De esta misma manera, se refiere incluso al lenguaje, tal y como se aprecia en El Crátilo de Platón, donde se pone de manifiesto a partir de Heráclito que, aunque todo nombre tenga que representar una esencia, ésta última estará siempre en un constante cambio ${ }^{34}$.

Teniendo en cuenta las ideas hasta aquí comentadas, se puede decir que Heráclito aborda el estudio de la naturaleza material y humana desde, lo que venimos a llamar en estas páginas, un paradigma de la complejidad, ya que sitúa como marco de referencia de su filosofía la complejidad de estas naturalezas, al hacer constantemente alusión a la oposición de sus elementos, así como al flujo continuo que las caracteriza. Estos son los motivos que nos hacen pensar que el adjetivo complejo se puede usar aquí tanto para definir su filosofía, repleta de dificultad y de variedad de interpretaciones, así como también para hacer referencia al marco de estudio desde el que, a mi entender, el autor la aborda: el paradigma de la complejidad.

La complejidad de la naturaleza material y humana se simboliza, también, en el pensamiento de Heráclito mediante la incorporación del elemento fuego $^{35}$ a modo de metáfora del mundo, no tanto creo como principio último arjé. De esta manera y a diferencia de otros filósofos presocráticos, quienes hablaron del agua, aire y/o apeiron, entre otros, como principios a partir de los que se originaban el resto de los elementos de la realidad, entiendo que Heráclito no tuvo la necesidad de incluirlos en su pensamiento porque, además, dichos principios hubiesen sido contradictorios con su defensa del cambio y del movimiento, tal y como se ha señalado en páginas anteriores. De acuerdo con esta interpretación, Spengler ${ }^{36}$ también niega la concepción del fuego en Heráclito como principio de todas las cosas y, más bien, lo entiende como una «pura metamorfosis transitoria como las demás, y sólo más apto estéticamente para representar la inquietud y el poder del cambio». Así, el fuego pasa a ser en Heráclito un símbolo del mundo; un reflejo del flujo perpetuo característico de la naturaleza material y humana si, además, tenemos en cuenta que ningún otro elemento podría reflejar tan fielmente, a través de sus movimientos ascendentes y descendentes, el desequilibrio constante causado por los continuos conflictos.

[33] Es necesario recordar aquí el inciso hecho en la primera nota al pie de página de este texto sobre el fuego como metáfora del mundo y no tanto como arjé.

[34] Sánchez Castro, L. C.: «La filosofía heraclítea interpretada por Platón» en Ideas y Valores LXI, 150, 2012, pp. 229-244.

[35] Gualdrón, M.: «Dios y fuego en la filosofía de Heráclito» en Saga 8, 2, 2003, pp. 9-18.

[36] Spengler, O.: op. cit., pp. 45-46.

THÉMATA. Revista de Filosofía, Nº52 julio-diciembre (2015) pp.: 179-197 doi: 10.12795/themata.2015.i52.10 
Este mundo, el mismo para todos, no lo hizo ninguno de los dioses ni de los hombres, sino que fue, es y será fuego siempre vivo, que se enciende y se apaga acompasadamente ${ }^{37}$.

Como se observa en esta cita textual, el fuego es, ciertamente, un elemento vivo, dinámico e inestable, fuente de vida y de muerte, naciendo la misma vida del fuego «[...] de la muerte de la materia que está siendo quemada y del oxígeno que lo alimenta» ${ }^{38}$. Además, es aquel elemento que mejor soporta y permanece todo posible cambio ${ }^{39}$, eterno e increado ${ }^{40}$, para el que"[...] cuando una determinada cantidad se extingue, se vuelve a encender en una parte proporcional» $»^{41}$. Por consiguiente, se puede decir que «el fuego trasciende toda oposición, está en todo sin serlo en efecto, y a su vez todo se presenta como el resultado de las transformaciones del fuego" ${ }^{42}$. Transformaciones a las que, por ejemplo, se hace alusión en los siguientes pasajes:

«Al transformarse el fuego según su primera medida nace el mar, y de la muerte del mar nacen, por un lado, la tierra y, por otro, el ardiente vapor, y, presumiblemente, de la muerte de éstos volverá a renacer el fuego hasta completar el ciclo cósmico, encendiéndose y apagándose del mismo modo que la vegetación se oculta [...] en otoño para volver a manifestarse» $[\ldots]$ en primavera ${ }^{43}$.

«El fuego todo lo cambia y por todo se cambia, del mismo modo que las mercancías por oro y el oro por las mercancías» ${ }^{44}$.

El fuego simboliza, por lo tanto, la oposición que caracteriza a la naturaleza material y humana, la cual se viene señalando en esas páginas como constituyente del paradigma de la complejidad en Heráclito, junto con la teoría del flujo perpetuo. Sin embargo, considero que es ahora ya el momento de matizar un poco más el concepto de oposición en este pensador presocrático con el fin de terminar de perfilar, de manera más completa, los diversos significados que puede adoptar en su filosofía. En general, creo que los dos siguientes son los sentidos del término oposición que se pueden encontrar en su obra:

1. Oposición entendida como antagonismo, contienda, contradicción, dificultad, discordancia, lucha... Causante del desorden y del desequilibrio en

[37] Gallero, J. L. y López, C. E.: op. cit., p. 97.

[38] Fernández Pérez, G.: op. cit., p. 153.

[39] Ibidem, pp. 89, 90.

[40] Ibídem, p. 91.

[41] Ibídem, p. 93.

[42] Ibidem, p. 123.

[43] Ibídem, p. 95.

[44] Gallero, J. L. y López, C. E.: op. cit., p. 55.

THÉMATA. Revista de Filosofía, №52 julio-diciembre (2015) pp.: 179-197

doi: 10.12795/themata.2015.i52.10 
la naturaleza material y humana. A este es al sentido de oposición al que nos hemos referido hasta el momento en estas páginas.

2. Oposición entendida como posibilitadora de la armonía, equilibrio, orden y solidaridad en la naturaleza material y humana. En Heráclito, y aquí está la concepción heraclitiana a la que todavía no se había hecho alusión en este texto, y que sigue haciendo evidente la complejidad de su pensamiento, es en la misma oposición, en la misma tensión, donde se encuentra la posibilidad de la armonía, convirtiéndose los mismos conflictos en las situaciones causantes del equilibrio y del orden del mundo material y humano. De esta manera, se pone de manifiesto en los siguientes extractos:

«[...] cabe hablar de una fricción creadora» ${ }^{45}$.

«[...] la tensión entre opuestos es la clave para avanzar en cualquier ámbito»"

«Ś́lo podrá adjetivar el desorden como destructor, de un modo aparente, quien no contemple el universo desde el punto de vista de la unidad de los opuestos» ${ }^{47}$.

«[...] un universo totalmente ordenado, sin liberar las tasas correspondientes de desorden, es tan inconcebible como un universo totalmente desordenado, en el que sería imposible cualquier tipo de permanencia y estabilidad ${ }^{48}$.

«La naturaleza, dicho de otro modo, mantiene su identidad visible gracias a una constante renovación invisible, posibilitada por la solidaridad de todos sus elementos»»49 .

«Por tanto, es preciso repensar el caos, entendido como fuego siempre-vivo, pues se trata de un concepto que no sólo no es contradictorio con la idea de ciclo, sino que además permite entender el carácter constructivo y no sólo destructivo de la guerra y el desorden cósmicos» $»^{50}$.

«El mundo de lo múltiple es un mundo-en-contienda, un gran campo de batalla, donde toda existencia nace del conflicto y vive en la tensión, pero no se trata de un conflicto únicamente destructivo, sino también productivo, puesto que la guerra, además de ser común, es padre de todas las cosas [...]. En este sentido, decir que la armonía visible del cosmos esconde una discordia invisible, sería tan cierto como decir que la discordia visible esconde una armonía invisible, puesto que la armonía es una consecuencia de la lucha misma: ajustamiento o justeza, un equilibrio que nace de la tensión entre los propios contrarios, pero nunca de su reconciliación ${ }^{51}$.

[45] Montes, Á. C.: op. cit., p. 48.

[46] Ibídem, p. 54.

[47] Fernández Pérez, G.: op. cit., p. 168.

[48] Ibidem, p. 170-171.

[49] Ibídem, p. 89.

[50] Ibidem, p. 153.

[51] Ibidem, p. 139.

THÉMATA. Revista de Filosofía, N52 julio-diciembre (2015) pp.: 179-197 doi: 10.12795/themata.2015.i52.10 
Las dos últimas citas que se incluyen más arriba nos llevan a introducir la idea de la guerra en el pensamiento de Heráclito, la cual mantiene aquí el mismo sentido que la continua oposición, tensión y conflictos que venimos comentando como característica esencial de la naturaleza material y humana. De esta manera, Fernández Pérez ${ }^{52}$ afirma que:

La guerra tiene un sentido simbólico en la filosofía de Heráclito [...] No parece que de los fragmentos conservados pueda desprenderse una apología del belicismo. Se trata de un proceso natural que comprende la totalidad del cosmos, generativo y regenerativo, en el que propiamente no hay vencedores ni vencidos, puesto que todos los contrarios son necesarios para que el mundo exista y mantenga su equilibrio.

Que la guerra tenga un carácter metafórico en el pensamiento de Heráclito, de acuerdo con el extracto anterior, entiendo que no supone contradicción alguna, puesto que lo que el filósofo pretende es seguir haciendo evidente la complejidad de la naturaleza, así como la permanencia de los conflictos en su cosmovisión del mundo. Conflictos a partir de los que es posible establecer la armonía, el orden y la justicia, siendo, por lo tanto, necesaria esta constante tensión para alcanzar cualquier conciliación posible ${ }^{53}$, tal y como se ha señalado en páginas anteriores de este mismo texto. Una conciliación que, necesariamente, ha de venir guiada por lo que Heráclito denomina $\log _{0}{ }^{54}$, que no es más que una razón que pasa a ser, según $\mathrm{Montes}^{55}$, el único medio del que dispone el ser humano para terminar con la discordia y alcanzar la paz. No obstante, es importante indicar que este logos no es una razón universal sino, simplemente, una razón común a todos los seres humanos (de la que gozan todas las personas) y que, en mi opinión, tiene en cuenta las diferencias existentes entre ellos. Se trata, por consiguiente, de una razón que parece tener carácter divino ${ }^{56}$, al convertirse en una ley necesaria que todo lo rige y que se encuentra en todo sujeto (aun teniendo en cuenta sus divergencias), de la misma manera que el fuego puede parecer tener también este mismo carácter divino, al simbolizar metafóricamente tanto el mundo en el que vivimos como la vida humana. Todo ello, nos hace pensar, entonces, en una armonía visible que se alcanza gracias a la misma tensión de los opuestos, a pesar de que Heráclito parece hablar de otra armonía invisible, resultado también de la continua discordia de los opuestos y regida por ella, situada más allá de lo sensible y que lo ubica, a mi entender, como un pensador más bien crítico con los sentidos. Podríamos interpretar esa armonía invisible, entonces, como esa posible armonía futura que

[52] Ibídem, p. 142-143.

[53] Ibidem, p. 157.

[54] Asensio, F. H.: «Heráclito y Parménides: maestros de sabiduría» en A Parte Rei 57, 2008, pp. 1-9. http://serbal.pntic.mec.es/AParteRei/

[55] Montes, Á. C.: op. cit., p. 55.

[56] Gualdrón, M.: op. cit.

THÉMATA. Revista de Filosofía, ${ }^{\circ} 52$ julio-diciembre (2015) pp.: 179-197 doi: 10.12795/themata.2015.i52.10 
siempre podrá tener lugar, hacia la que se seguirá avanzando mientras se vayan superando las tensiones y los conflictos, y que siempre seguirá siendo invisible en la medida en que la perdurabilidad de los conflictos sea eterna, siendo, por consiguiente, imperecedera la necesidad de seguir afrontándolos, siguiendo alcanzando, con ello, situaciones de armonía, justicia y orden. Es decir, mientras la perdurabilidad de las tensiones sea eterna, eterna será la necesidad de seguir afrontándolas para alcanzar la armonía, así como eterna será, también, esa posible armonía futura que es invisible. Nos damos cuenta entonces que la armonía invisible podría hace alusión, en mi opinión, a la posibilidad de seguir alcanzando situaciones de justicia en el futuro, a poder ser mejoradas respecto a las anteriores, teniendo en cuenta la perdurabilidad de los conflictos y, con ello también, la necesaria perdurabilidad de seguir afrontándolos con el fin de ir mejorando y perfeccionando, poco a poco, las situaciones de orden, equilibrio y justicia social y humana, aunque ni que decir tiene que dichas situaciones nunca podrán ser perfectas en términos absolutos porque siempre seguirán teniendo lugar tensiones y discordias que podrán ser superadas.

Hasta aquí un conjunto de rasgos y características que reflejan la complejidad del pensamiento de Heráclito, interpretado desde lo que en estas páginas hemos venido a llamar el paradigma de la complejidad.

\section{Los conflictos interculturales en relación con el paradigma de la complejidad de Heráclito}

La cosmovisión del mundo de Heráclito es de gran utilidad para interpretar los conflictos, en el caso de estas páginas los conflictos interculturales ${ }^{57}$, que tienen lugar entre los seres humanos en las diferentes sociedades. El rasgo de la oposición que tan fielmente defiende en su concepción de la naturaleza

[57] Hablaremos de conflictos interculturales en el marco de las teorías de la interculturalidad. Respecto a esta última, cabe decir que la interculturalidad se entiende como aquella corriente que se esfuerza por poner en conexión a las distintas culturas, favoreciendo y facilitando el aprendizaje entre ellas y creando espacios comunes de encuentro interculturales para la convivencia (Cf. Panikkar, R.: Paz e interculturalidad. Una reflexión filosófica. Barcelona: Herder, 2006). Muy diferente es, por consiguiente, la interculturalidad de otras corrientes terminológicas que hablan, por ejemplo, de «inclusión» (Cf. Habermas, J.: La inclusión del otro: estudios de teoría política. Barcelona: Paidós, 1999) y/o de multiculturalidad. En relación con esta última, diremos que supone, más bien y en mi opinión, la visualización de la suma de todas las culturas (esto es, somos sociedades que contienen muchas culturas en un mismo lugar), aunque sin ponerlas en conexión, sino, simplemente, interpretándolas como estancos separados, con valores y estilos de vida diferentes que debemos aceptar, aunque no, necesariamente reconocer, pudiendo llegar, entonces y en los casos más extremos, al relativismo. A diferencia de ello, la interculturalidad no acepta todo de todas las culturas, sino que, sobre todo, ejerce una fuerte crítica sobre aquello que pueda atentar, en cada una de ellas, a la integridad humana, permitiendo transformaciones en los valores culturales y construyendo, como decíamos, espacios comunes de encuentro interculturales en los que todos y todas se sientan igualmente reconocidas.

THÉMATA. Revista de Filosofía, №52 julio-diciembre (2015) pp.: 179-197 doi: 10.12795/themata.2015.i52.10 
material y humana, característica fundamental de su paradigma de la complejidad, se convierte en una pieza básica que complementa y ayuda a clarificar a la perfección la concepción que, especialmente y desde los estudios para la paz $^{58}$, se viene defendiendo de las situaciones conflictivas. Así y de la misma manera que señala Heráclito, las investigaciones para la paz afirman la existencia continua de conflictos tanto en el mundo material como en el interior de los seres humanos, hasta el extremo de señalar su carácter inherente a la naturaleza humana ${ }^{59}$. Tanto es así que se dice que las personas no podemos vivir sin conflictos ni a nivel interno ni a nivel intersubjetivo, ya que, en primer lugar, interiormente manifestamos de manera continua nuestra indecisión ante decisiones que debemos adoptar y que nos hacen dudar sobre cuál pueda ser nuestra mejor opción. En este sentido, esta indecisión se hace patente a la hora de abordar decisiones sencillas, como, por ejemplo, qué beber y/o qué comer en un momento concreto, o cómo vestirnos para una determinada ocasión especial, así como, también, cuando afrontamos decisiones que implican una mayor reflexión y que, por lo tanto, suponen una mayor dificultad, como, por ejemplo, la que yo siento al analizar de qué modo puedo estructurar estas páginas, cómo actuar en un momento específico y/o cuál pueda ser el mejor camino para hacer frente a una delicada situación. En segundo lugar, intersubjetivamente, también vivimos continuos conflictos con las otras personas que nos rodean, bien por cuestiones de la vida cotidiana, laborales, familiares y/o de amistad, entre otras. Lo que sí que es cierto es que los conflictos están y se dan y que no podríamos vivir sin ellos porque, de lo contrario, pareceríamos estar de acuerdo con todo lo que tenemos y muy poco, por lo tanto, podrían avanzar las relaciones sociales y humanas. Cuando un conflicto surge es porque hay alguna cosa que nos disgusta y el hecho de ser consciente de ese desagrado es, a mi entender, el primer paso para reclamar un cambio, una transformación de eso que nos incomoda. Otra cosa será, tal y como veremos posteriormente, de qué manera se gestiona esa transformación y qué carácter adopta el conflicto en cada caso.

Entonces, estamos poniendo encima de la mesa aquí ese rasgo de la continua oposición que señalaba Heráclito, aunque ejemplificado en este lugar con conflictos que se dan en la vida cotidiana. De acuerdo con esta idea, a nivel intersubjetivo otra tipología de conflictos está teniendo cada vez más lugar. Me refiero a los conflictos interculturales, que son a los que se quiere prestar

[58] Cabe recordar aquí que, para la elaboración de este trabajo, se sigue la línea de investigación de la filosofía para la paz de la Cátedra UNESCO de Filosofía para la Paz de la Universitat Jaume I de Castellón, la cual se enmarca en el conjunto de investigaciones llevadas a cabo por los estudios para la paz, aunque, en este caso, desde la reflexión filosófica.

[59] Lederach, J. P.: Preparing for peace. Conflict transformation across cultures. New York, Syracuse University Press, 1995; Muñoz, F. A.: «Qué son los conflictos» en Molina Rueda, B. y Muñoz, F. A.: Manual de Paz y Conflictos. Granada: Universidad de Granada, 2004, pp. 143-170; París Albert, S.: Filosofía de los conflictos. Una teoría para su transformación pacífica. Barcelona: Icaria, 2009.

THÉMATA. Revista de Filosofía, ${ }^{\circ} 52$ julio-diciembre (2015) pp.: 179-197 doi: 10.12795/themata.2015.i52.10 
mayor atención en estas páginas desde la corriente de la interculturalidad, y que suceden cuando se producen encuentros entre personas procedentes de diversas culturas. Encuentros que son de gran actualidad en nuestras sociedades cuando los movimientos migratorios son, absolutamente, constantes, haciendo visibles los contactos culturales en cualquier parte del mundo. Estos conflictos me permiten seguir haciendo hincapié en el rasgo de la continua oposición defendida en el pensamiento de Heráclito, evitando referirme, de esta manera y en este estudio, a cualquier otro conflicto intersubjetivo y/o interno que también pueden tener lugar, aunque los rasgos mencionados se puedan aplicar a todos ellos. Esta explícita intención me posibilita mantenerme en la corriente de la intersubjetividad que, además, tan fehacientemente ha sido analizada desde los estudios que venimos realizando en la filosofía para la paz de la Cátedra UNESCO de Filosofía para la Paz de la Universitat Jaume I de Castellón, conservando la unión con los estudios más generales y clásicos para la paz ${ }^{60}$.

En la línea de lo que venía a decirnos Heráclito, podemos afirmar que los conflictos están y se dan; que generamos continuamente situaciones conflictivas, tal y como ya decíamos más arriba, siendo, absolutamente, necesario aprender a convivir con ellas ${ }^{61}$. Para alcanzar este objetivo, nuestra principal tarea deberá ser la de asumir y comprender la conflictividad humana, acostumbrándonos a ella como un rasgo distintivo de la complejidad de la naturaleza humana. Esto quiere decir, por ejemplo, que como sujetos debemos habituarnos a vivir conflictos interculturales, con personas procedentes de otras culturas, sin tener ningún miedo a ello. Esta es la razón por la que será importante educarnos para estar preparados a encontrarnos con esta clase de conflictos interculturales, siendo muy conscientes de que vamos a vivirlos a lo largo de nuestra vida. Solo de esta manera podremos ser sabedores de nuestra conflictividad, de su carácter inevitable, así como de las posibles alternativas para su transformación. Estos son algunos de los objetivos principales de los estudios para la paz, en general, y de la filosofía para la paz, en particular.

Las ideas que vengo comentando en las líneas anteriores nos hacen pensar en cuán complejas somos las personas; complejidad, que interpretada desde lo que hemos venido en llamar paradigma de la complejidad en $\mathrm{He}$ ráclito, se caracteriza no sólo por los constantes conflictos que vivimos, sino también por los cambios continuos que experimentamos los sujetos. De acuerdo con esta afirmación y al hilo de la teoría del perpetuo flujo heraclitiana, podemos señalar, entonces, que los seres humanos estamos cambiando también

[60] Martínez Guzmán, V.: Filosofía para hacer las paces. Barcelona: Icaria, 2001; Martínez Guzmán, V.: Podemos hacer las paces: Reflexiones éticas tras el 11-S y el 11-M. Bilbao: Ed. Desclée de Brouwer, 2005.

[61] París Albert, S.: Filosofía de los conflictos cit.; París Albert, S.: «Filosofía, transformación de conflictos y paz» en Comins Mingol, I. y París Albert, S.: Investigación para la paz: estudios filosóficos. Barcelona: Icaria, 2010, pp. 89-104; París Albert, S.: «Naturaleza humana y conflicto: Un estudio desde la Filosofía para la Paz» en Eikasia. Revista de Filosofía 50, 2013, pp. 107-116.

THÉMATA. Revista de Filosofía, N52 julio-diciembre (2015) pp.: 179-197 doi: 10.12795/themata.2015.i52.10 
continuamente, de tal manera que, por ejemplo, muchas de las situaciones que hoy apreciamos como conflictos, podríamos no percibirlas igual con el tiempo y/o gran parte de los acuerdos que hoy consideramos válidos para hacer frente a muchas situaciones conflictivas, podríamos no entenderlos de este modo en el futuro. Además, a este hecho se añade la gran variedad de respuestas que todo conflicto puede tener ${ }^{62}$, dependiendo en gran medida, tanto su validez como su eficacia, de los individuos que los están viviendo y de en qué momento de sus vidas los están sobrellevando. A pesar de ello y en relación con los conflictos interculturales, es necesario tener en cuenta, también, otro aspecto relativo a la procedencia cultural, ya que esta última puede influir, muy y mucho, en la elección que hacemos de las respuestas para afrontar una determinada situación conflictiva, pues de por sí, por ejemplo, lo que quienes hemos sido educados en España entendemos por conflicto no tiene por qué serlo para quienes han sido educados en otros lugares del mundo, convirtiéndose, en muchas ocasiones, estas diferencias en causas principales de los conflictos interculturales. En mi opinión y de acuerdo con las investigaciones para la paz, es este un criterio que no podemos olvidar y que pone de manifiesto la importancia del contexto social cuando hablamos de conflictos ${ }^{63}$, especialmente de conflictos interculturales, teniendo que recordar, en el marco de la teoría del flujo perpetuo de Heráclito, que nuestra percepción de las situaciones conflictivas puede variar según en qué parte del mundo nos encontremos, incluso pudiéndose transformar ésta si una vez criados en lugar de la tierra, nos trasladamos a otro, comenzando allí una nueva vida y compartiendo los valores del nuevo lugar.

Como se puede observar, las últimas líneas del párrafo anterior nos permiten recuperar el debate sobre las diferencias, sobre las que tanto se ha discutido, siendo un tema muy recurrente a la hora de hablar sobre los conflictos interculturales. Me gustaría hacer aquí un pequeño paréntesis para indicar que la línea de investigación a partir de la que se escribe este texto, la cual se enmarca en la filosofía para la paz, tal y como se viene mencionando, toma como punto de partida el carácter positivo de las diferencias, viendo no sólo su aceptación, sino su reconocimiento como un paso necesario para la transformación de las situaciones conflictivas $^{64}$. Se tratará, entonces, de no negar las diferencias, sino, simplemente, de reconocerlas, entendiéndolas y buscando posibles conexiones entre ellas a partir de las que crear nuevos espacios comunes de encuentro interculturales que favorecerán, sin ningún lugar a dudas, la transformación de los conflictos.

[62] Fisas, V.: Cultura de paz y gestión de conflictos. Barcelona: Icaria, 1998; Lederach, J. P.: Preparing for peace cit.

[63] Fisas, V.: op. cit.; Fisher, R., Kopelman, E. y Schneider, A. K.: Más allá de Maquiavelo: Herramientas para afrontar conflictos, Barcelona: Granica, 1996; Lederach, J. P.: Preparing for peace cit.

[64] Fisher, R., Kopelman, E. y Schneider, A. K.: op. cit.

THÉMATA. Revista de Filosofía, №52 julio-diciembre (2015) pp.: 179-197 doi: 10.12795/themata.2015.i52.10 
Volvamos ahora al tema que nos ocupa. Estamos hablando de flujo perpetuo y constantes conflictos como rasgos que nos ayudan a entender la complejidad humana, al estilo de lo que venía a decirnos Heráclito en su cosmovisión del mundo. Sin embargo, me falta añadir algo más respecto a esta continua oposición de Heráclito reflejada aquí con los constantes conflictos que vivimos, en este caso estamos diciendo conflictos interculturales. Me refiero ahora a las dos nociones de este concepto que ya hemos señalado en el apartado anterior y que, también, se encuentra en la interpretación de los conflictos que se trabaja en los estudios para la paz. Hablamos de:

1. Oposición entendida como antagonismo, contienda, contradicción, dificultad, discordancia, lucha... Esta visión del término es equiparable a la manera de interpretar los conflictos en la década de los cincuenta, cuando eran considerados situaciones dañinas, causantes de destrucciones materiales y físicas, y con las que era necesario terminar tan pronto como fuese posible ${ }^{65}$.

2. Oposición entendida como posibilitadora de la armonía, equilibrio, orden y solidaridad en la naturaleza material y humana. Esta visión del término es equiparable a la manera de interpretar los conflictos a partir de la década de los noventa, momento en el que se considera que los conflictos son imprescindibles para visualizar muchas de las tensiones que existen en las relaciones interpersonales, así como son posibilitadores de cambios, al hacer posible tomar conciencia de qué está pasando y poner en marcha, al mismo tiempo, acciones para transformar dichas situaciones ${ }^{66}$, tal y como ya se ha dejado entrever en páginas anteriores. Ni que decir tiene que no me estoy refiriendo en ningún momento a los conflictos como situaciones positivas o negativas en sí mismas, sino, simplemente, como situaciones que tienen lugar en la vida y que hay que afrontar. Lo que, en este caso, sería positivo o negativo son los medios usados para su transformación, dando lugar, entonces, a una transformación violenta si los medios son violentos y/o a una positiva si los medios son pacíficos. De nuevo, observamos lo que ya decíamos anteriormente en estas páginas, haciendo alusión a que lo realmente importante es cómo se gestiona esa transformación para que los conflictos, en este trabajo decimos interculturales, adopten un carácter u otro.

La segunda noción de oposición es la que se identifica, plenamente, con la terminología de la transformación de los conflictos, que, como se habrá podido observar, es la que vengo usando en estas páginas en lugar de hacer uso de otras nomenclaturas posibles, siendo, al mismo tiempo, en la que seguiré profundizando en el siguiente apartado de este trabajo, mientras continuamos buscando las conexiones entre los conflictos interculturales, su transformación pacífica y la interpretación de la naturaleza material y humana de Heráclito.

[65] Lederach, J. P.: Preparing for peace cit.

[66] Ibídem; París Albert, S.: Filosofía de los conflictos cit.

THÉMATA. Revista de Filosofía, N52 julio-diciembre (2015) pp.: 179-197 doi: 10.12795/themata.2015.152.10 


\section{El paradigma de la complejidad heraclitiana y la transforma- ción pacífica de los conflictos interculturales}

La terminología de transformación de conflictos ha venido siendo usada a lo largo de este texto siempre que he tenido que referirme a la regulación de los conflictos interculturales. Ha sido sólo en el último párrafo cuando se ha especificado, de forma más concreta, el uso de esta nomenclatura junto con el adjetivo pacífico, hablando así de una transformación pacífica de los conflictos ${ }^{67}$. Ni que decir tiene que he procedido de esta manera para dejar constancia de que la transformación de un conflicto (añadimos intercultural) puede hacerse tanto por medios violentos como por medios pacíficos, siendo, claro está, estos últimos los que se defienden en estas páginas. De este modo, se sostiene que debemos hacer un gran esfuerzo por aprender a transformar nuestros conflictos interculturales por medios pacíficos, cambiando las costumbres en las que hayamos podido ser educados; poniendo nuestras energías en el aprendizaje de otras alternativas; y ocupando nuestro tiempo en cultivarnos en estos otros hábitos pacíficos, por muy difícil y costoso que ello pueda parecernos ${ }^{68}$. Tal y como afirmamos desde la filosofía para la $\mathrm{paz}^{69}$, el uso de medios pacíficos para la regulación de los conflictos interculturales es una alternativa más que tenemos los seres humanos, visualizando, así, nuestra responsabilidad sobre la decisión de usar estos medios y/o la violencia. Es, por consiguiente, en esta decisión donde se manifiesta la libertad que nos permite escoger entre opciones diversas, según cuál de ellas podamos considerar como la más adecuada en cada momento.

El verbo transformar significa, si tomamos en consideración su raíz etimológica del latín transformare, "dar otro aspecto a algo o a alguien» ${ }^{70}$. De ahí que la expresión conceptual transformación pacífica de los conflictos pueda representar una oportunidad para dar un «nuevo aspecto» a las relaciones conflictivas a través de afrontar las tensiones que en ellas aparecen, creando, al mismo tiempo, nuevos objetivos que permitirán el mantenimiento de estas relaciones en el futuro. Sin embargo, creo que es importante clarificar ahora que una cosa es la terminología y otra muy diferente lo que metodológicamente se esconde detrás de esta nomenclatura. Pues si bien es cierto que la transformación de los conflictos interculturales, ese nuevo aspecto que podemos darles, se puede hacer por medios violentos y/o pacíficos, siendo estos últimos por los que abogamos en este texto como ya se ha dicho, la metodología de la transformación pacífica de los conflictos tiene unas implicaciones muy específicas y

[67] Me gustaría matizar, un vez más, que abogo en este texto por la transformación pacífica de los conflictos tanto en lo que a su terminología como a su metodología se refiere.

[68] Cascón Soriano, P.: Educar en y para el conflicto. Barcelona: Universitat Autònoma de Barcelona, 2001.

[69] Martínez Guzmán, V.: Filosofía para cit.; Martínez Guzmán, V.: Podemos hacer las paces cit. [70] Moliner, M.: Diccionario del uso del español. Madrid: Gredos, 1997 (versión en CD-ROM).

THÉMATA. Revista de Filosofía, ${ }^{\circ} 52$ julio-diciembre (2015) pp.: 179-197 doi: 10.12795/themata.2015.i52.10 
concretas. En este sentido y como metodología de regulación de las situaciones conflictivas, surge a partir de la década de los noventa en el marco de los estudios para la paz, dejando al margen a la resolución ${ }^{71}$ y a la gestión $n^{72}$ de conflictos, que son las dos metodologías que se habían venido usando con anterioridad. Así mismo, la transformación pacífica de conflictos promueve una serie de características novedosas respecto a las situaciones conflictivas (aplicadas aquí al caso de la interculturalidad), entre las que quiero destacar las dos siguientes $\left.{ }^{73}: 1\right)$ Supone una noción de las situaciones conflictivas interculturales como situaciones a revalorizar para el desarrollo de las relaciones humanas, interpretándolas como posibilitadoras de armonía, justicia y orden, siempre y cuando éstas sean reguladas por medios pacíficos. 2) Diseña un conjunto de medios pacíficos a tener en cuenta durante el proceso de la transformación de conflictos interculturales, favorables a su transformación pacífica, entre los que se puede resaltar: la cooperación, el uso de poderes en positivo, la comunicación no violenta, el reconocimiento recíproco, el empoderamiento y la recuperación de los sentimientos.

Estamos poniendo encima de la mesa, entonces, una metodología que nos ofrece la posibilidad de abordar las situaciones conflictivas, en este caso interculturales, por medios pacíficos, dándoles un nuevo aspecto, al estilo de las transformaciones constantes que sufría el fuego en la cosmovisión de la naturaleza material y humana de Heráclito. No obstante y siguiendo con la relación de esta concepción de los conflictos interculturales con Heráclito, cabe decir que nunca esta metodología permitirá poner fin a todos los conflictos, pues, a pesar de que podrá favorecer la consecución de acuerdos comunicativos, siempre nuevos conflictos interculturales podrán tener lugar, los cuales, nuevamente, tendremos que transformar, haciendo posible esa insistencia constante para lograr la armonía invisible que ya comentábamos en el apartado anterior. Incluso, un conflicto previamente tratado podría volver a resurgir en el futuro bien por las mismas causas bien por causas diferentes que lo traen otra vez a colación ${ }^{74}$.

[71] La resolución de conflictos es la metodología que aparece en la década de los cincuenta, cuando nos encontramos en los comienzos de los estudios para la paz. Esta metodología entiende a los conflictos como situaciones negativas con las que hay que terminar a toda costa para poder evitar los daños materiales y humanos que causan.

[72] La gestión de conflictos es la metodología que surge en la década de los setenta, una vez ya se han asentado las investigaciones para la paz. Por primera vez, esta metodología promueve una visión positiva de los conflictos, aunque sigue poniendo el énfasis en las consecuencias destructivas y dañinas que éstos causan.

[73] Lederach, J. P.: Preparing for peace cit.

[74] Lederach, J. P.: Building peace. Sustainable reconciliation in divides societies. Washington: United States Institute for Peace, 1998.

THÉMATA. Revista de Filosofía, N52 julio-diciembre (2015) pp.: 179-197 doi: 10.12795/themata.2015.i52.10 
Recuperábamos arriba la armonía invisible y decíamos armonía hacia la que tendemos a medida que vamos transformando pacíficamente los conflictos interculturales. Por hacer un inciso, me recuerda esta idea de la armonía invisible, tal y como se interpreta en este texto, a la teoría de la paz imperfec$t a^{75}$, que tanta trascendencia ha tenido en los estudios para la paz. Desde un punto de vista histórico, la paz imperfecta nos indica que nunca podremos hablar de una paz perfecta, sino de momentos de paz imperfectos que iremos viviendo y perfeccionando a medida que vayamos transformando pacíficamente conflictos, teniendo muy en cuenta el aprendizaje ya obtenido en experiencias previas de regulación de situaciones conflictivas. Será este un proceso de perfeccionamiento de la paz guiado por la razón humana (el logos heraclitiano), común a todos los seres humanos, pero solidario con las diferencias de todo tipo, entre ellas, las culturales, aunque basándose en un punto de vista intercultural. Este es el motivo por el que la razón humana (no sólo lógica, sino también emocional) beneficiará los procesos de transformación pacífica de los conflictos interculturales, al poner, también, en práctica algunos de los medios de esta metodología anteriormente mencionados. Me estoy refiriendo, especialmente, a la cooperación y al reconocimiento recíproco. Medios que propician, sin ninguna duda, la interculturalidad, al fundamentar sus acciones en la solidaridad, la tolerancia y el respeto.

\section{Conclusiones}

El pensamiento complejo de Heráclito se ha convertido en una herramienta útil para seguir con la interpretación de los conflictos, en este caso interculturales, y de la metodología para su transformación pacífica que venimos investigando en la Cátedra UNESCO de Filosofía para la Paz de la Universitat Jaume I de Castellón. Sobre todo y en esta conceptualización, me gustaría destacar los rasgos de la oposición y del devenir, los cuales son rasgos distintivos de lo que aquí hemos considerado el paradigma de la complejidad de Heráclito.

Para terminar quisiera decir que este texto se puede considerar como un primer estudio de una serie de trabajos que tengo la intención de seguir elaborando con la finalidad de abordar, en cada uno de ellos, las posibles conexiones existentes entre la interpretación de los conflictos de la filosofía para la paz y otros pensadores, como, por ejemplo, Hegel y/o Nietzsche. Todo ello con el firme convencimiento de que la filosofía puede sernos muy valiosa a lo hora de indagar en los seres humanos, en sus relaciones y en los conflictos con los que diariamente se encuentran.

[75] Muñoz, F. A.: La paz imperfecta. Granada: Universidad de Granada, 2001.

THÉMATA. Revista de Filosofía, Nº52 julio-diciembre (2015) pp.: 179-197 doi: 10.12795/themata.2015.i52.10 
